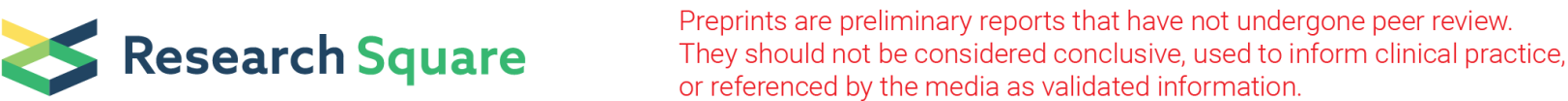

\section{Chorionic villus-derived mesenchymal stem cell- mediated autophagy promotes proliferation and invasiveness of trophoblasts under hypoxia by activating the JAK2/STAT3 signalling pathway}

\section{Yijing Chu}

Affiliated Hospital of Medical College Qingdao University

Chongyu Yue

Affiliated Hospital of Medical College Qingdao University

Wei Peng

Affiliated Hospital of Medical College Qingdao University

Weiping Chen

Affiliated Hospital of Medical College Qingdao University

\section{Yan Zhang}

Affiliated Hospital of Medical College Qingdao University

\section{Guifang He}

Affiliated Hospital of Medical College Qingdao University

Changchang Liu

Affiliated Hospital of Medical College Qingdao University

Ning Zhang

Affiliated Hospital of Medical College Qingdao University

Jing Li

Affiliated Hospital of Medical College Qingdao University

Ling Liu

Affiliated Hospital of Medical College Qingdao University

\section{Guoqiang Gao}

Affiliated Hospital of Medical College Qingdao University

Ke Yao

Affiliated Hospital of Medical College Qingdao University

Yan Li

Affiliated Hospital of Medical College Qingdao University

Xiaoyu Hu

Affiliated Hospital of Medical College Qingdao University

\section{Xiaofei Wang}

Affiliated Hospital of Medical College Qingdao University 


\section{Rendong Han}

the affiliated hospital of qingdao university

\section{Yuxiang Huang}

allcare biomedical department

Yuanhua Ye ( $\nabla$ yeyuanhua8067@126.com )

Affiliated Hospital of Medical College Qingdao University https://orcid.org/0000-0002-0517-0557

\section{Research}

Keywords: Chorionic villous derived mesenchymal stem cells, trophoblasts, autophagy, JAK2/STAT3 signalling pathway

Posted Date: May 8th, 2020

DOI: https://doi.org/10.21203/rs.3.rs-25663/v1

License: (c) (i) This work is licensed under a Creative Commons Attribution 4.0 International License. Read Full License 


\section{Abstract}

Objectives Trophoblast dysfunction during pregnancy is fundamentally involved in preeclampsia. The aim of this study was to understand how human chorionic villous mesenchymal stem cells (CV-MSCs) operate in regulation of trophoblast function.

Materials and Methods We treated trophoblasts with CV-MSC supernatant under hypoxic conditions, and transcriptome and pathway analyses of trophoblasts were performed. Western blotting and PCR analysis were used to examine the JAK2, STAT3 and autophagy associated protein expression levels in trophoblasts.

Results The CV-MSC supernatant treatment markedly enhanced proliferation, invasion and autophagy. The RNA-seq revealed JAK2/STAT3 signalling as an upstream regulator, and STAT3 mRNA and protein levels increased during CV-MSC treatment. Inhibition of JAK2/STAT3 signalling reduced autophagy, survival and invasion of trophoblasts even in the presence of CV-MSCs, and blocking autophagy did not affect STAT3 activation in trophoblasts treated with CV-MSCs. Importantly, overexpression of STAT3 increased the levels of autophagy in trophoblasts; thus, it regulated positively autophagy in hypoxic trophoblasts. Human placental explants also proved our finding, in which STAT3 was activated and LC3B-II levels were increased by CV-MSC treatment.

Conclusions Our data suggest that CV-MSC-dependent activation of JAK2/STAT3 signalling is a prerequisite for upregulation of autophagy in trophoblasts.

\section{Introduction}

As a special kind of hypertensive disorder occurring during pregnancy, preeclampsia (PE) is one of the prime reasons of maternal and perinatal morbidity and mortality ${ }^{1-3}$. Although increasing numbers of researchers have worked to understand the molecular mechanisms of PE progression, the underlying mechanisms responsible for the pathogenesis and triggering factors of PE remain elusive. It has been suggested that the proliferative and invasive capacity of extravillous trophoblasts (EVTs) is essential for the development of the placenta; however, failed spiral artery remoulding and insufficient oxygen and nutrient supply cause EVT invasion dysfunction, which ultimately results in placental dysfunction and adverse pregnancy outcomes ${ }^{4-6}$. In addition, aberrant placental implantation can cause a state of elevated oxidative stress and hypoxia, which lead to inflammation and antiangiogenic protein release ${ }^{7}$.

Since autophagy degrades aggregates of damaged organelles or misfolded proteins and manipulates the "regeneration" of cells, it is a vital process of cell survival under stress and inflammation 8,9 . Due to the hypoxic and inflammatory responses associated with $\mathrm{PE}$, placental trophoblasts in individuals with $\mathrm{PE}$ have more reliance on autophagy for survival compared to normal cells $s^{10,11}$. Autophagy protects syncytiotrophoblasts from apoptosis, infection, and inflammation in the human placenta ${ }^{12,13}$, many 
questions remain regarding the exact aetiology and precise pathogenic mechanisms ensuring autophagic flux.

Chorionic villi, which form the innermost layer of the placenta and are critical components of the maternal-foetal interface, continue growing throughout pregnancy to enrich the foetus with nutrients and blood supply from the mother ${ }^{5,14}$. Placental pathologies, such as PE and foetal growth restriction are related with the dysplasia or dysfunction of placental chorionic villi; thus, it also crucial for placental development ${ }^{4,15}$. Foetal CV-MSCs are crucial in normal placental development; as multipotent stromal cells, CV-MSCs are detached from chorionic villi ${ }^{16}$. Many studies have suggested that CV-MSCs can promote angiogenesis through paracrine effects and potentially participate in placental pathologies in vascular system, including PE as well as foetal growth restriction. However, there is currently no information on the roles of CV-MSCs in the proliferative and invasive capacity of EVTs.

Here, the effects of CV-MSC conditioned medium (CM) on the proliferative and invasive capacities of the trophoblast lines JAR, JEG-3 and HTR-8 were studied. In addition to increasing proliferative and invasive capacity, CV-MSC CM significantly enhanced autophagy in the three trophoblast lines under hypoxic conditions. Transcriptome analyses showed considerable upregulation of the inflammatory response and the signalling pathway of IL-6/JAK/signal transducer and activator of transcription 3 (STAT3) in trophoblasts; JAK2/STAT3 was putatively recognized as a positive upstream regulator of autophagy under hypoxic conditions.

\section{Methods And Materials}

\section{Cell culture}

Placentas were obtained from full-term births after caesarean section and age-matched placentas from severe preeclampsia after caesarean section with parental permission. All CV-MSCs were used at passages 3-6 in this study. In line with the ethical protocols of the Affiliated Hospital of Qingdao University, China, every procedure was conducted accordingly. In brief, the foetal portion of the placenta was minced into approximately $1-\mathrm{mm}^{3}$ slices and then rinsed in PBS; the aim of that is to remove all blood. The tissues were treated with trypsin (0.25\%) and collagenase (0.1\%) (type I; Sigma-Aldrich, St. Louis, MO). Afterward, they were subjected to incubation at a temperature of thirty-seven degrees Celsius for thirty minutes. After filtration through a $100-\mu \mathrm{m}$ nylon filter and centrifugation, we plated the cells on culture plates in a stem cell culture medium, which contained Stem Cell Basic Medium (Dakewe Biotech Co., Guangzhou, China) and 5\% UltraGRO ${ }^{\mathrm{TM}}$ (Helios, USA). An incubator with thirty-seven degrees Celsius and $5 \% \mathrm{CO}_{2}$ was used to culture the primary cells.

The obtained JAR, JEG-3, and HTR-8 cells from the Type Culture Collection China Centre were subjected to culture and then used for experiments. DMEM/F12 containing $10 \%$ FBS was used to culture all three trophoblast lines in an incubator with thirty-seven degrees Celsius and $5 \% \mathrm{CO}_{2}$. The medium was changed when the confluency reached $50 \%$. The cells were subjected to incubation for a set time at a 
temperature of thirty-seven degrees Celsius; the humidified atmosphere of the incubator contains $93 \% \mathrm{~N}_{2}$, $5 \% \mathrm{CO}_{2}$, and $2 \% \mathrm{O}_{2}$ (Invivo2 Hypoxia Workstation, Ruskinn Technology, Leeds, West Yorkshire, UK). For each experiment, these cells were subjected to culture triplicately.

\section{CV-MSCs identification}

The flow cytometry (with obtained antibodies from eBioscience, San Diego, CA, including CD34, CD105, CD73, CD90, CD44, CD45, IG1 and HLA-DR) was used to examine the expression of cellular markers in CV-MSCs (passage 3); these markers consist of positive markers (CD44, CD73, CD90 and CD105) and negative markers (CD34, CD45, CD146, IG1 and HLA-DR).

Moreover, CV-MSCs from normal placenta or severe preeclampsia placenta are able to change into osteoblasts as well as adipocytes by differentiation; therefore, we assessed this ability. CV-MSCs cultured in 6-well plates were grown to approximately $70-80 \%$ confluence. Then, the CV-MSCs were subjected to culture in a differentiation medium (osteogenic or adipogenic) (Gibco, Carlsbad, CA) for three weeks. Alizarin red S was used to stain the CV-MSCs to verify osteoblast differentiation; for adipocyte differentiation, oil red $O$ was selected.

\section{CM preparation}

When CV-MSCs isolated from the placentas reached $80 \%$ confluence, we replaced the medium with DMEM/F12 (Gibco, Carlsbad, CA) lacking FBS and cultured the cells for an additional $24 \mathrm{~h}$. After a twelveminute centrifugation $(1,200 \times \mathrm{g})$, the medium underwent a filtration through a filter $(0.22 \mu \mathrm{m})$ (Millipore, Billerica, MA). Then, it was collected for subsequent experiments. The DMEM/F12 medium lacking FBS were used as control $\mathrm{CM}$.

\section{Antibodies as well as reagents}

We bought anti-microtubule-associated protein LC3 and BECN1 (beclin1, an initiator of autophagosomes) antibodies from Sigma-Aldrich Corporation, which is located in St. Louis, USA. Cell Signalling Technology (Danvers, MA, USA) provided the following antibodies: anti-STAT3, anti-pSTAT3, anti-JAK2, anti-pJAK2, anti-P62, anti-MMP2, and anti-MMP9. For cryptotanshinone (a STAT3 inhibitor), 3-methyladenine (an autophagy inhibitor) (3MA, $10 \mathrm{mM}$ ) and bafilomycin $\mathrm{A} 1$ (Baf $\mathrm{A} 1$, an inhibitor of autophagosomelysosome fusion), they were purchased from MedChem Express (NH, USA). A STAT3 plasmid was purchased from Shanghai Genechem Co., Ltd. Recombinant human interleukin-6 (IL-6) and IL-6 antibodies, were bought from R\&D Systems. 


\section{RNA-seq and gene set enrichment analysis (GSEA)}

TRIzol Reagent ( $1 \mathrm{ml}$ ) (Thermo Fisher Scientific) was used to collect samples; these samples were then placed at a temperature of eighty degrees Celsius below zero. We prepared libraries based on the instructions for an Illumina TruSeq RNA Sample Prep Kit and carried out sequencing on a MiSeq instrument. RSEM software was used to analyze the experimental data from Annoroad Gene Technology Co., Ltd. (Beijing, China). Labelled as no. SRR9943697-no. SRR9943702, all these data (RNA-seq) can be accessed at Sequence Read Archive (SRA).

c2.cp.v5.1.symbols of the gene set database were used to carry out GSEA of the RNA-seq data when the fold change data were input into GSEA software (Broad Institute) from the analysis of differential expression.

\section{Transmission electron microscopy}

7.4-pH PBS with glutaraldehyde (2.5\%) was used to fix the trophoblasts; then it was stored at $4{ }^{\circ} \mathrm{C}$ for more than two hours. $1 \% \mathrm{OsO}_{4}$ was used to post-fix the cells for two hours; then, they were dehydrated in ethyl alcohol and propanone. Thereafter, we imbedded the cells in epoxy resin. After cutting ultrathin sections in a size of 50-60 nm, these sections were positioned on uncoated copper grids; $3 \%$ lead citrateuranyl acetate was used to stain ultrathin sections. JEM-1200EX transmission electron microscope was used to obtain the images (JEOL, Tokyo, Japan).

\section{Quantitative real-time PCR (RT-PCR)}

CV-MSC CM was used to treat JAR, JEG-3, and HTR-8 cells for $24 \mathrm{~h}$. Subsequently, we extracted total RNA from the trophoblasts using TRIzol Reagent (Takara, Japan). Then, we used a reverse transcription kit (Invitrogen) to synthesize complementary DNA. Gene-specific TaqMan probes (Applied Biosystems), as well as master mix (Thermo Fisher Scientific), were used to carry out quantitative RT-PCR; the operations obeyed the instructions made by the manufacturer. The expression of target gene was normalized to GAPDH expression. We used the TaqMan probes, including probes for IL6 (Hs00985639_m1), STAT3 (Hs00374280_m1), GAPDH (Hs02786624_g1), and leukaemia inhibitory factor (LIF; Hs01055668_m1). Three separate reactions were performed for each marker.

\section{Trophoblast invasion assay}


Cells from the three lines of trophoblasts $\left(5 \times 10^{5}\right.$ cells) were seeded in Transwell chambers in 24-well plates (Corning, NY, USA). $50 \mu$ of a 1:10 dilution of Matrige ${ }^{\mathrm{TM}}$ matrix was used to cover the pore membranes $(8 \mu \mathrm{m})$ in the twenty four-well plates. The trophoblasts were cultured on the membranes for $12 \mathrm{~h}$. One of two types of the medium was added into the lower chamber: CV-MSC CM supplemented with $10 \%$ FBS or normal culture medium supplemented with $10 \%$ FBS (control). Transwell assays were performed while culturing the trophoblasts without FBS for $24 \mathrm{~h}$. Next, $4 \%$ paraformaldehyde was used to fix all cells. Trophoblasts that penetrated through the membranes were analysed. The cells that migrated through the membrane were counted in high-magnification fields. Every experiment was performed three times.

\section{Cell proliferation analysis}

We added cells from the three trophoblast lines to ninety six-well plates (density: 5,000 cells per well), then cultured these cells, and measured trophoblast proliferation daily via CCK-8 reagent (Thermo Fisher Scientific, MA, USA). We added the CCK-8 reagent to each well, and the trophoblasts were cultured for an additional $1.5 \mathrm{~h}$. Then, colorimetric assays were performed by measuring the absorbance (optical density [OD] value) of each well in a microplate reader (wavelength: $450 \mathrm{~nm}$ ). Growth curves were determined in three independent experiments.

\section{Western blotting}

Trophoblasts were lysed on ice for 12 min by using RIPA buffer (Sigma, St. Louis, MO, USA). After centrifugation at $12,000 \times \mathrm{g}$, the cell lysates were treated with LDS Sample Buffer. Then, SDS-PAGE was used to separate the protein mixtures, which were then shifted from the gel to a membrane of polyvinylidene fluoride (PVDF) (Bio-Rad, Hercules, CA). Next, 5\% skim milk was used to block the membrane.

Subsequently, primary rabbit monoclonal antibodies against human JAK2, pJAK2, STAT3, pSTAT3, MMP2, MMP9, LC3, BECN1 and P62 (1:1,000 dilution) or $\beta$-actin (same dilution; Proteintech, Chicago, IL) were used to incubate the blocked membrane. Then, secondary antibodies were used to incubate the membrane (1:1,000; CST, Danvers, MA). We detected and quantified the protein-antibody complexes using a chemiluminescence detection system (Bio-Rad, Hercules, CA).

\section{Placental explant culture}

Within a 10-min operation, all placentas were collected, which were then treated within 30 min and closely examined for any seeable abnormalities. After being thoroughly rinsed in PBS for 3 times to get rid of 
maternal blood, placental villous tissue was dissected into $8-\mathrm{mm}^{3}$ pieces of tissue $(2 \mathrm{~mm} \times 2 \mathrm{~mm} \times 2$ $\mathrm{mm}$ ). DMEM/F12 (4 ml per well) with $1 \%$ penicillin/streptomycin and amphotericin B (Gibco, Carlsbad,

$\mathrm{CA}$ ) was used to culture the placental explants in six-well dishes (Corning) in a hypoxic incubator for $48 \mathrm{~h}$ at $37^{\circ} \mathrm{C}$; the oxygen content was $2 \%$. After CV-MSC CM treatment for $24 \mathrm{~h}$, PBS was used to rinse the explants; after that, they were frozen in liquid nitrogen.

\section{Immunohistochemistry}

$4 \%$ paraformaldehyde was used to fix the human term placental explants $(n=5)$ for $60 \mathrm{~min}$. We embedded the tissues in paraffin, sliced them into 4- $\mu \mathrm{m}$ sections, and deparaffinized them. Then, the slides were boiling in 6.0-pH sodium citrate buffer $(10 \mathrm{mM})$ for $7 \mathrm{~min}$ at a temperature of one hundred and twenty degrees Celsius for antigen retrieval. Hydrogen peroxide was used to block endogenous peroxidase for $10 \mathrm{~min}$. We washed the slides three times for $5 \mathrm{~min}$ each with TBS, which contained $0.05 \%$ Tween 20 (TBS/T; Merck; Darmstadt, Germany); later, these slides were incubated with monoclonal antiSTAT3 antibodies $(1: 200)$ and anti-P62 antibodies $(1: 1,000)$ for $12 \mathrm{~h}$ at a temperature of four degrees Celsius. Diluted biotinylated secondary antibodies were used to incubate the sections for twenty min at a temperature of thirty-seven degrees Celsius. We visualized the target proteins via fresh DAB solution; then, we utilized haematoxylin as a tissue counterstain. Through an optical microscope (Olympus FV500, Tokyo, Japan), the expression of the target proteins was assessed by two observers independently. Via image-Pro Plus 5.1, the area, as well as the intensity of staining in five random regions (200x magnification), were analyzed; thus, the expression level of proteins was assessed.

\section{Statistical analysis}

One-way analysis of variance (ANOVA) or two-tailed Student's t-tests was used to carry out statistical analyses; data were reported in the form of the mean \pm standard deviation from more than three experiments, which were independently performed. If the $P$ value was less than 0.05 , it demonstrated a significant difference.

\section{Results}

Growth, invasion, and autophagy of trophoblasts was promoted by CV-MSCs under hypoxic conditions

First, we isolated CV-MSCs from healthy and severe preeclampsia placentas and identified the multidirectional differentiation ability and surface marker expression of the CV-MSCs (Fig. S1A-B). Next, we used CCK-8 assays, transwell assays and transmission electron microscopy to examine the proliferation, invasion, and autophagy, respectively, of trophoblasts treated with or without CV-MSC CM under hypoxic conditions in order to evaluate the influence of CV-MSCs on these cells (Fig. 1A-C). The CV- 
MSC CM-treated trophoblasts exhibited significantly higher proliferation than the untreated cells in hypoxic condition (all $\mathrm{P}<0.001$ ). Based on the results of the transwell assays, CV-MSC CM increased the number of penetrated trophoblasts after $24 \mathrm{~h}$ under hypoxic culture system $(\mathrm{P}<0.001)$. To verify whether the CV-MSC from severe PE placenta have the same trophoblasts promoting effects, we examined the proliferation and invasion of trophoblasts treated with CM of PE-CV-MSC. The PE-CV-MSC CM-treated trophoblasts exhibited slightly higher proliferation in JEG-3 and HTR-8 cells than the untreated cells (all $P>0.05)$, and three lines trophoblasts invasion ability were not increased after treatment of PE-CV-MSC $\mathrm{CM}$ (Fig. S2A-B). These results indicated that the function of CV-MSCs in PE placenta may be impaired by the pathological condition.

To investigate the influences of CV-MSCs on trophoblasts autophagy, electron microscopy analysis was performed, and the results revealed that there were more autophagosomes in CV-MSC CM-treated trophoblasts than in control trophoblasts (Fig. 1C). In accordance with these observations, via western blotting, the BECN1 expression and LC3-II/LC3-I ratio became higher while the p62 level became lower, in the three lines of trophoblasts after treatment with CV-MSC CM under hypoxic conditions (Fig. 1D). Increased MMP2 and MMP9 levels were also observed in cells treated with CV-MSC CM (Fig. 1D). Thus, CV-MSCs promoted trophoblast proliferation, invasion, and autophagy.

\section{Global transcriptome characterization of trophoblasts regulated by CV-MSCs under hypoxic condition}

To better understand the changes in JEG-3 cells that occurred after CV-MSC treatment, we compared the transcriptomes of JEG-3 cells cultured for $48 \mathrm{~h}$ with or without CV-MSC CM under hypoxic condition. Through RNA-seq, we found 336 upregulated and 859 downregulated genes changed more than two folds (Fig. 2 A). Moreover, Gene Set Enrichment Analysis (GSEA) was used to compare the changed signalling pathways in trophoblasts regulated by the CV-MSCs treatment with control trophoblasts. From the results, the up-regulation of cytokine signaling pathways was proved; JAK2/STAT3 signalling was identified as one of the pathways which showed the most significant upregulation in trophoblasts treated with CV-MSCs (Fig. 2 B). In summary, our analyses revealed CV-MSCs upregulated proliferation, invasion,

and autophagy of trophoblast cells, while IL-6/JAK/STAT3 signaling was upregulated significantly in this process.

\section{The JAK2/STAT3 signalling pathway was activated in trophoblasts cultured with CV-MSC CM}

We further examined how the CV-MSC treatment influenced on JAK2/STAT3 signalling pathway of trophoblasts under hypoxic condition. CV-MSC CM-treated trophoblasts exhibited approximately 3-fold higher STAT3 mRNA levels than untreated trophoblasts after $48 \mathrm{~h}$ (Fig. 2C). IL-6 and LIF, which are activators of STAT3, were dramatically upregulated in CV-MSC CM-treated trophoblasts compared with untreated trophoblasts (Fig. 2C). Consistent with the mRNA data, JAK2, STAT3, pJAK2, and pSTAT3 
protein levels were significantly elevated in CV-MSC CM-treated trophoblasts compared to control trophoblasts (Fig. 2D). To evaluate the JAK2/STAT3 signalling pathway and autophagy in trophoblasts treated with PE-CV-MSC CM, we examined the mRNA and protein expression of STAT3 and autophagy related markers in trophoblasts under hypoxic condition (Fig. S2C-D). There were no significant activation of STAT3 signalling or increased autophagy activity in trophoblasts after PE-CV-MSC CM treatment, and the mRNA levels of IL- 6 and LIF were stable in CV-MSC CM-treated trophoblasts compared with untreated trophoblasts.

Since we studied all the expriments under hypoxic conditions, we then examined the JAK2/STAT3 signalling, autophagy and MMPs expression in trophoblasts cultured under normal oxygen tension and hypoxic condition. Our results showed that hypoxia slightly increased the autophagy activity and MMP2/MMP9 expression, and the JAK2/STAT3 signalling pathways were not activated in trophoblasts (Fig. S3A). The results reveal that the JAK2/STAT3 signalling pathway could be activated by CV-MSC treatment in trophoblasts under hypoxic condition, and the CV-MSCs in healthy donors' placenta exhibit higher activity compared to PE patients' placenta.

\section{The JAK2/STAT3 signalling pathway mediated increased trophoblast autophagy under hypoxic conditions}

After confirming that CV-MSCs activated the autophagy through JAK2/STAT3 signalling pathway in trophoblasts, we subsequently investigated whether this signalling pathway regulated the autophagy of trophoblasts. Trophoblasts were stimulated with the STAT3 activator IL- 6 and the specific STAT3 inhibitor cryptotanshinone. As a bioactive compound, cryptotanshinone is detached from the roots of Salvia miltiorrhiza Bunge, and it blocks STAT3 phosphorylation at Tyr70 $5^{17}$. When $8 \mathrm{mM}$ cryptotanshinone was present, the p-STAT3/STAT3 ratio in JEG-3 cells was almost fifty percent lower than it was absent (Fig. $2 \mathrm{E})$. Also, in the presence of cryptotanshinone (8 $\mathrm{mM})$, the expression of autophagy-related proteins in CV-MSC CM-treated cells was investigated. We discovered that p-STAT3, p-JAK2 as well as BECN1 levels and the LC3-II/LC3-I ratio dropped; moreover, p62 levels were higher in the three trophoblast lines in the presence than in the absence of $8 \mathrm{mM}$ cryptotanshinone (Fig. 2F). We studied the same trends in cells treated with cryptotanshinone (8 mM) and IL-6 (Fig. 2F).

To determine whether the IL-6 present in CV-MSC CM is responsible for the observed effects, we examined the JAK2/STAT3 signalling and autophagy related proteins in trophoblasts with IL- 6 antibody and CV-MSC CM treatment using western blotting (Fig. S3B). The results showed that the neutralization of IL-6 in CM by antibodies attenuated the activation of STAT3 signalling and autophagy of trophoblasts by CV-MSC CM. Thus, the results showed that STAT3 acted as positive regulation of CV-MSC-induced autophagy and IL-6 secreted by CV-MSC may act as an activator of STAT3 signalling in trophoblasts. 
CV-MSCs promoted trophoblast growth and metastasis through the JAK2/STAT3 signalling pathway

To further investigate how JAK2/STAT3 signalling pathway influenced trophoblast regulation by CVMSCs, we analysed trophoblast proliferation and invasion after cryptotanshinone treatment. Cells were cultured in CV-MSC CM or IL-6 for $24 \mathrm{~h}$; then cryptotanshinone ( $8 \mathrm{mM}$ ) was used to culture them for $24 \mathrm{~h}$; CCK-8 assay investigates the changes in cell growth (Fig. 3A). IL-6 and CV-MSC CM individually increased cell viability, while cryptotanshinone reversed the enhancing effects of IL-6 and CV-MSC CM. Transwell assays were also performed; we studied the same trends in cells when cryptotanshinone and IL- 6 or CVMSC CM was treated (Fig. 3B). These results demonstrated that CV-MSCs accelerated trophoblast proliferation as well as invasion through the JAK2/STAT3 signalling pathway.

\section{Inhibition of autophagy decreased trophoblast growth and invasion even under conditions of JAK2/STAT3 signalling pathway activation}

To assess whether autophagy inhibition is connected to decreased growth and invasion in trophoblasts, we treated trophoblasts cultured with or without CV-MSC CM for $24 \mathrm{~h}$ with an autophagy inhibitor (3-MA, $10 \mathrm{nM}$ ), and the western blot results confirmed that 3-MA reduced the LC3-II/LC3-I ratio as well as MMP2 and MMP9 levels in trophoblasts, even CV-MSC CM-treated trophoblasts (Fig. 3C). pJAK2 and pSTAT3 expression were increased in the three trophoblast lines treated with CV-MSC CM with or without 3-MA (Fig. 3C). Then, the proliferation and invasion abilities of trophoblasts treated with 3-MA and CV-MSC CM were tested using CCK-8 and transwell assays. Even in the presence of CV-MSC CM, 3-MA suppressed the growth and invasion of three trophoblast lines (Fig. 3D-E).

To further determine the role of CV-MSC on the autophagic flux of trophoblasts, the BECN1, P62 and LC-3 II/LC3-I ratio were compared in the trophoblasts treated with CV-MSC CM, in the presence of bafilomycin A1 (Baf A1) (Fig. S3C). LC-3 II/LC3-I ratio, BECN1 and P62 levels wre significantly increased in trophoblasts treated with Baf A1. In contrast, the LC-3 II/LC3-I ratio was not significantly different between Baf A1 treated and untreated cells, when they were co-treated with CV-MSC CM. Moreover, the BECN1 and P62 levels were increased significantly between Baf A1 treated and untreated cells, when they were cotreated with CV-MSC CM. These results indicated that the CV-MSC CM may activate the trophoblasts autophagy through inducing autophagic flux, and inhibiting autophagy can constrain the proliferation as well as the invasion ability of trophoblasts with or without CV-MSC CM treatment.

\section{STAT3 regulated autophagy in trophoblasts under hypoxic conditions}

To examine if STAT3 activation increases trophoblast autophagy, viability, and invasion, we transfected trophoblasts with a STAT3 plasmid, then overexpressed STAT3, and examined the mRNA expression of STAT3 in trophoblasts (Fig. 4A, P<0.001). A CCK-8 assay indicated that the viability of cells was significantly increased by STAT3 plasmid transfection compared to empty control vector transfection 
(Fig. 4C, P<0.001). Consistent with the CCK-8 assay results, the transwell assay results suggested that STAT3-overexpressing trophoblasts showed higher invasion ability than control trophoblasts, as determined by the number of penetrated cells (Fig. 4D, P<0.001).

We further examined autophagy-related protein expression and MMP2/MMP9 levels in STAT3 plasmidtransfected trophoblasts (Fig. 4B). The western blotting results showed that P62 levels were decreased in these cells, whereas MMP2/MMP9 and LC3 II levels were enhanced, suggesting that STAT3 positively regulates trophoblast autophagy.

\section{CV-MSC-mediated STAT3 activation increased autophagy in placental explants}

After that, data from trophoblasts was compared with placental explant cultures, which are in vivo models in which villous trophoblasts remain in a natural environment under more physiologically relevant conditions than those in traditional cell cultures. The human placental explants were incubated in DMEM/F12 and then in medium containing CV-MSC CM or IL-6 with or without the STAT3 inhibitor cryptotanshinone for $48 \mathrm{~h}$, respectively. Western blot assays were used to determine the p-JAK2, pSTAT3, and LC3 II levels in the placental explants. While P62 levels were decreased in the CV-MSC CM- and IL-6treated placental explants compared to the control explants, p-JAK2, pSTAT3, and LC3 II levels showed obvious increasing trends (Fig. 5A). Additionally, immunohistochemistry showed increased STAT3 staining intensity of villous cytotrophoblast cytoplasm and decreased P62 staining intensity of villous cytotrophoblast cytoplasm in CV-MSC CM-treated placental explants (Fig. 5B) compared to control explants. Interestingly, syncytiotrophoblast nuclei remained unstained in the cryptotanshinone-treated group, suggesting that CV-MSCs activate STAT3 and enhance autophagy in villous cytotrophoblasts.

Taken together, these data suggest that CV-MSC mediates JAK2/STAT3 activation and autophagic activity in cultured placental explants.

\section{Discussion}

It is vital for placentation to precisely regulate autophagy, and some evidence suggests that autophagy in the placenta is impaired during $\mathrm{PE}^{18}$. Shigeru Saito et al. proposed an autophagy inhibition hypothesis for the pathophysiology of PE: at the early stage of pregnancy, abnormal placentation is induced by autophagy inhibition in trophoblasts; thus, the poor placentation leads to chronic and severe hypoxia in the placenta. Furthermore, severe hypoxia in the placenta exaggerates impairment of trophoblasts as well as inflammation and dysregulation of homeostasis, resulting in a vicious cycle ${ }^{19}$. Although autophagy status changes are observed in preeclamptic placentas, the mechanisms that modulate autophagy and autophagy-related biological changes in placentas remain unknown. Here, we provide evidence that CV- 
MSC-mediated JAK2/STAT3 signalling pathway activation is the regulatory mechanism of autophagic activity in human villous trophoblasts and promotes the proliferation and invasion of trophoblasts.

Many studies have proved the therapeutic potential of mesenchymal stem cells (MSCs), multipotent cells isolated from various human tissues, in tissue repair; this therapeutic potential is attributable to the capacity of these cells to undergo multipotent differentiation and to modulate immune responses ${ }^{20-22}$. CV-MSCs that are capable of self-renewal and differentiation and that exhibit immunoregulatory properties can be isolated from human term placenta, which is abundant and commonly discarded after delivery $^{23}$. Some studies have revealed roles of CV-MSCs in the regulation of endothelial cells, macrophages, natural killer cells, and dendritic cells ${ }^{24-27}$. Due to the outstanding properties of CV-MSCs, they are potential alternative MSCs which can be applied in cell-based therapy ${ }^{28}$, but research on the functions of these cells has been limited. We investigated how human CV-MSCs influenced trophoblasts under hypoxic conditions and found that CV-MSCs regulate autophagy to influence the proliferation and invasion ability of trophoblasts through the JAK2/STAT3 signalling pathway.

As a potential transcription factor, STAT3 has a mediative function in the interaction between extracellular signalling molecules (e.g., cytokines and growth factors) and polypeptide receptors on the surface of cells ${ }^{29}$. STAT3 keeps a basal level in cells, whereas it sharply increases via self-activation if stimulation occurs. In addition, STAT3 is constitutively activated in a majority of malignant tumours with high invasiveness and autophagy levels, suggesting that STAT3 works as an oncogene ${ }^{30}$. Recent literature has also explored how STAT3 regulates autophagy and has emphasized that STAT3 affects autophagy in various ways ${ }^{31}$. Some studies have indicated that STAT3 seems to inhibit autophagy in some kinds of tumours and infectious diseases ${ }^{32,33}$, but others have shown that STAT3 activation can significantly enhance autophagy in some blood tumours and autoimmune and inflammatory diseases $^{34,35}$. STAT3, responding to a variety of autophagy inducers, is a well-known stress-responsive nuclear factor ${ }^{31}$. However, it is still not completely reasoned how specific stimuli drive STAT3 signalling and how it regulates autophagy.

Trophoblast dysfunction, which leads to cellular ischaemia and hypoxia, oxidative stress and vascular endothelial injury, is involved in hypertensive diseases during pregnancy ${ }^{36}$. Current treatment strategies of PE focus on improving placental microcirculation and preventing maternal and foetal complications ${ }^{3}$. However, there is no treatment addressing the aetiology of trophoblastic dysfunction despite the importance of trophoblast autophagy for placentation and PE is clear. It has been described in previous studies that autophagy, a defense mechanism in healthy cells, is driven by p53 during human trophoblast differentiation ${ }^{37}$. Autophagy activation has been shown to be efficacious in helping to prevent dysfunction of normal cells and as a targeted therapeutic strategy in multiple preclinical models; for example, activation of autophagy protects hepatocytes from chemical-induced hepatotoxicity ${ }^{19}$. These findings could provide novel insight into potential PE therapies. 
To sum up, it was described in our study that CV-MSCs promote the proliferation as well as invasion of trophoblasts under hypoxic conditions in vitro, perhaps at least partially due to activation of JAK2/STAT3 signalling, which upregulates trophoblast autophagy. For the symptoms of PE usually occur after 20 weeks of gestation, the condition is first observed long after the failure of trophoblast invasion (usually completed before 8-10 weeks of gestation). Furthermore, our results emphasize the urgent need for early diagnosis and treatment of placental abnormalities. However, it is still unclear how CV-MSCs influence other types of placenta-based cells or even in vivo; thus, it remains to study whether CV-MSCs affect them similarly. Further study on how STAT3 regulates autophagy would be important for the thorough insight into PE physiology and a novel therapeutic strategy for PE, such as CV-MSC treatment.

\section{Conclusions}

Our data suggest that CV-MSCs promote the survival and function of trophoblasts under hypoxic conditions by activating JAK2/STAT3 signalling with trophoblast autophagy upregulation. Our findings provide new potential therapeutic or preventive approaches for placenta-related disorders during pregnancy.

\section{Abbreviations}

CV-MSCs: Chorionic villous mesenchymal stem cells; JAK2: Janus-activated kinase 2; STAT3: Signal transducer and activator of transcription 3; PE: Preeclampsia; EVTs: extravillous trophoblasts; CM: Conditioned medium; MMP: Matrix metalloproteinases; IL-6: Interleukin-6; PBS: Phosphate-buffered saline; FBS: Foetal bovine serum.

\section{Declarations}

\section{Ethics approval and consent to participate}

The study was approved by the Ethics Committee of the Qingdao University Affiliated Hospital. All participants provided a written informed consent.

\section{Consent for publication}

Not applicable.

\section{Availability of data and materials}


The datasets used and/or analyzed during the current study are available from the corresponding author on reasonable request.

\section{Competing interests}

The authors declare no potential conflicts of interest.

\section{Acknowledgements}

All funders had no role in the design of the study and collection, analysis, and interpretation of data and in writing the manuscript. We thank the Department of Obstetrics and Gynaecology, the Affiliated Hospital of Qingdao University, Qingdao, China, for providing tissue samples.

\section{Authors' contributions}

Y.Y. and Y.H. designed the project. Y.C., C.Y., W.P., Y.Z. W.C. and R.H.developed the experiments. G.H., C.L., N.Z., J.L., G.G. and L.L. analyzed the data. Y.J., and Y.L. wrote the paper. K.Y., X.W. and X.H. performed differentiations and analyzed the experiments.

\section{Funding Sources}

This study was supported by the Clinical Medicine+X Project of the Affiliated Hospital of Qingdao University.

\section{References}

1. Ramos JGL, Sass N, Costa SHM, Preeclampsia. Rev Bras Ginecol Obstet. 2017;39:496-512. doi:10.1055/s-0037-1604471.

2. El-Sayed AAF, Preeclampsia. A review of the pathogenesis and possible management strategies based on its pathophysiological derangements. Taiwan J Obstet Gynecol. 2017;56:593-8. doi:10.1016/j.tjog.2017.08.004.

3. Folk DM. Hypertensive Disorders of Pregnancy: Overview and Current Recommendations. Journal of Midwifery Women's Health. 2018;63:289-300. doi:10.1111/jmwh.12725.

4. Ridder A, Giorgione V, Khalil A, Thilaganathan B, Preeclampsia. The Relationship between Uterine Artery Blood Flow and Trophoblast Function. Int J Mol Sci 20, doi:10.3390/ijms20133263 (2019). 
5. Kusuma GD, Abumaree MH, Pertile MD, Kalionis B in Preeclampsia Methods in Molecular Biology Ch. Chapter 19, 247-266 (2018).

6. Shah S, Gupta A. Hypertensive Disorders of Pregnancy. Cardiol Clin. 2019;37:345-54. doi:10.1016/j.ccl.2019.04.008.

7. Mol BWJ, et al. Pre-eclampsia. The Lancet. 2016;387:999-1011. doi:10.1016/s0140-6736(15)000707.

8. Mizushima N, Komatsu M. Autophagy: renovation of cells and tissues. Cell. 2011;147:728-41. doi:10.1016/j.cell.2011.10.026.

9. Ho TT, et al. Autophagy maintains the metabolism and function of young and old stem cells. Nature. 2017;543:205-10. doi:10.1038/nature21388.

10. Nakashima A, et al. Autophagy is a new protective mechanism against the cytotoxicity of platinum nanoparticles in human trophoblasts. Scientific reports 9, doi:10.1038/s41598-019-41927-2 (2019).

11. Nakashima A, et al. Current Understanding of Autophagy in Pregnancy. Int J Mol Sci 20, doi:10.3390/ijms20092342 (2019).

12. Zhang J, et al. Deletion of the Type IV Secretion System Effector VceA Promotes Autophagy and Inhibits Apoptosis in Brucella-Infected Human Trophoblast Cells. Curr Microbiol. 2019;76:510-9. doi:10.1007/s00284-019-01651-6.

13. Zenclussen AC, Hutabarat M, Wibowo N, Huppertz B. The trophoblast survival capacity in preeclampsia. Plos One 12, doi:10.1371/journal.pone.0186909 (2017).

14. Akolekar R, Beta J, Picciarelli G, Ogilvie C, D'Antonio F. Procedure-related risk of miscarriage following amniocentesis and chorionic villus sampling: a systematic review and meta-analysis. Ultrasound in Obstetrics Gynecology. 2015;45:16-26. doi:10.1002/uog.14636.

15. Bosco Becerra C, et al. Placental Hypoxia Developed During Preeclampsia Induces Telocytes Apoptosis in Chorionic Villi Affecting The Maternal-Fetus Metabolic Exchange. Curr Stem Cell Res Ther. 2016;11:420-5. doi:10.2174/1574888x10666150202144855.

16. Paulraj B, Kannaiyan J, Muthukutty P, Iqbal MDT. Villous chorion: A potential source for pluripotentlike stromal cells. Journal of Natural Science Biology Medicine 8, doi:10.4103/0976-9668.210011 (2017).

17. Qi P, et al. Cryptotanshinone Suppresses Non-Small Cell Lung Cancer via microRNA-146a-5p/EGFR Axis. Int J Biol Sci. 2019;15:1072-9. doi:10.7150/ijbs.31277.

18. Nakashima A, et al. Autophagy regulation in preeclampsia: Pros and cons. J Reprod Immunol. 2017;123:17-23. doi:10.1016/j.jri.2017.08.006.

19. Nakashima A, et al. Role of autophagy in oocytogenesis, embryogenesis, implantation, and pathophysiology of pre-eclampsia. J Obstet Gynaecol Res. 2017;43:633-43. doi:10.1111/jog.13292.

20. Carvalho AÉS, Sousa MRR, Alencar-Silva T, Carvalho JL, Saldanha-Araujo F. Mesenchymal stem cells immunomodulation: The road to IFN-y licensing and the path ahead. Cytokine Growth Factor Rev. 2019;47:32-42. doi:10.1016/j.cytogfr.2019.05.006. 
21. Knaän-Shanzer S. Concise Review: The Immune Status of Mesenchymal Stem Cells and Its Relevance for Therapeutic Application. Stem cells. 2014;32:603-8. doi:10.1002/stem.1568.

22. $10.1155 / 2016 / 9156731$

Huang Y, et al. Effects of Human Umbilical Cord Mesenchymal Stem Cells on Human Trophoblast Cell Functions In Vitro. Stem cells international 2016, 9156731, doi:10.1155/2016/9156731 (2016).

23. Abumaree $\mathrm{MH}$, et al. Phenotypic and Functional Characterization of Mesenchymal Stem Cells from Chorionic Villi of Human Term Placenta. Stem Cell Reviews Reports. 2012;9:16-31. doi:10.1007/s12015-012-9385-4.

24. Abumaree $\mathrm{MH}$, et al. Preconditioning human natural killer cells with chorionic villous mesenchymal stem cells stimulates their expression of inflammatory and anti-tumor molecules. Stem Cell Res Ther 10, doi:10.1186/s13287-019-1153-9 (2019).

25. Abomaray FM, et al. Human Chorionic Villous Mesenchymal Stem Cells Modify the Functions of Human Dendritic Cells, and Induce an Anti-Inflammatory Phenotype in CD1 + Dendritic Cells. Stem Cell Reviews Reports. 2014;11:423-41. doi:10.1007/s12015-014-9562-8.

26. Basmaeil YS, et al. Human chorionic villous mesenchymal stem/stromal cells protect endothelial cells from injury induced by high level of glucose. Stem Cell Res Ther 9, doi:10.1186/s13287-0180984-0 (2018).

27. Abumaree MH, et al. Human Placental Mesenchymal Stem Cells (pMSCs) Play a Role as Immune Suppressive Cells by Shifting Macrophage Differentiation from Inflammatory M1 to Antiinflammatory M2 Macrophages. Stem Cell Reviews Reports. 2013;9:620-41. doi:10.1007/s12015013-9455-2.

28. Umezawa A, et al. Amnion-derived cells as a reliable resource for next-generation regenerative medicine. Placenta. 2019. doi:10.1016/j.placenta.2019.06.381.

29. Abroun S, et al. STATs: An Old Story, Yet Mesmerizing. Cell J. 2015;17:395-411.

30. Wake MS, Watson CJ. STAT3 the oncogene - still eluding therapy? FEBS J. 2015;282:2600-11. doi:10.1111/febs.13285.

31. You L, et al. The role of STAT3 in autophagy. Autophagy. 2015;11:729-39. doi:10.1080/15548627.2015.1017192.

32. Hu F, et al. Docetaxel-mediated autophagy promotes chemoresistance in castration-resistant prostate cancer cells by inhibiting STAT3. Cancer letters. 2018;416:24-30. doi:10.1016/j.canlet.2017.12.013.

33. Zhang Y-G, et al. Intestinal epithelial HMGB1 inhibits bacterial infection via STAT3 regulation of autophagy. Autophagy, 1-19, doi:10.1080/15548627.2019.1596485 (2019).

34. Chang L, Feng $X$, Gao W. Proliferation of rheumatoid arthritis fibroblast-like synoviocytes is enhanced by IL-17-mediated autophagy through STAT3 activation. Connect Tissue Res. 2018;60:358-66. doi:10.1080/03008207.2018.1552266.

35. Larrue C, et al. Oncogenic KIT mutations induce STAT3-dependent autophagy to support cell proliferation in acute myeloid leukemia. Oncogenesis 8, doi:10.1038/s41389-019-0148-9 (2019). 
36. Lee CQE, et al. Integrin a2 marks a niche of trophoblast progenitor cells in first trimester human placenta. Development 145, doi:10.1242/dev.162305 (2018).

37. Gauster $\mathrm{M}$, et al. Downregulation of $\mathrm{p} 53$ drives autophagy during human trophoblast differentiation. Cell Mol Life Sci. 2017;75:1839-55. doi:10.1007/s00018-017-2695-6.

\section{Figures}
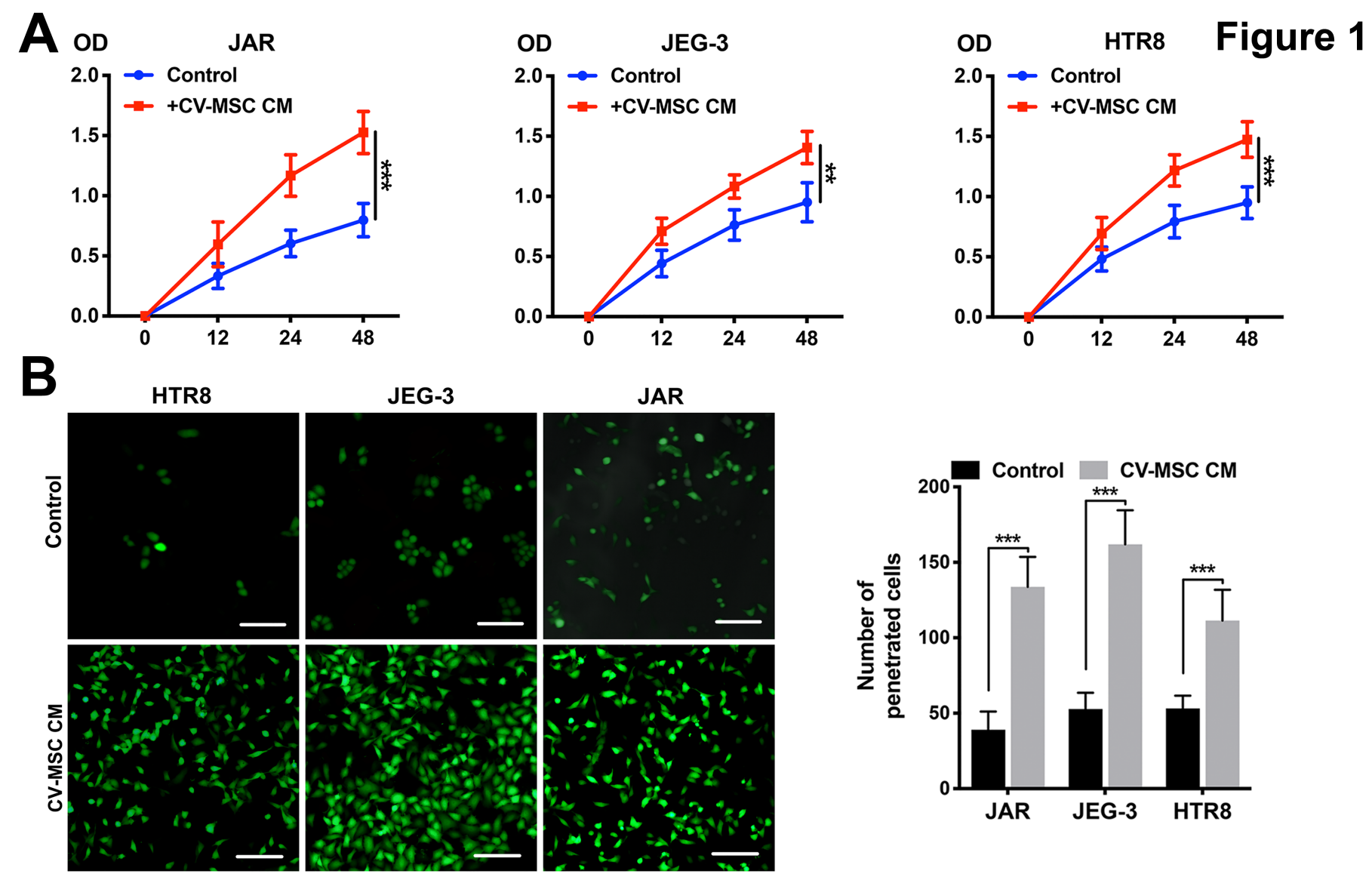

C

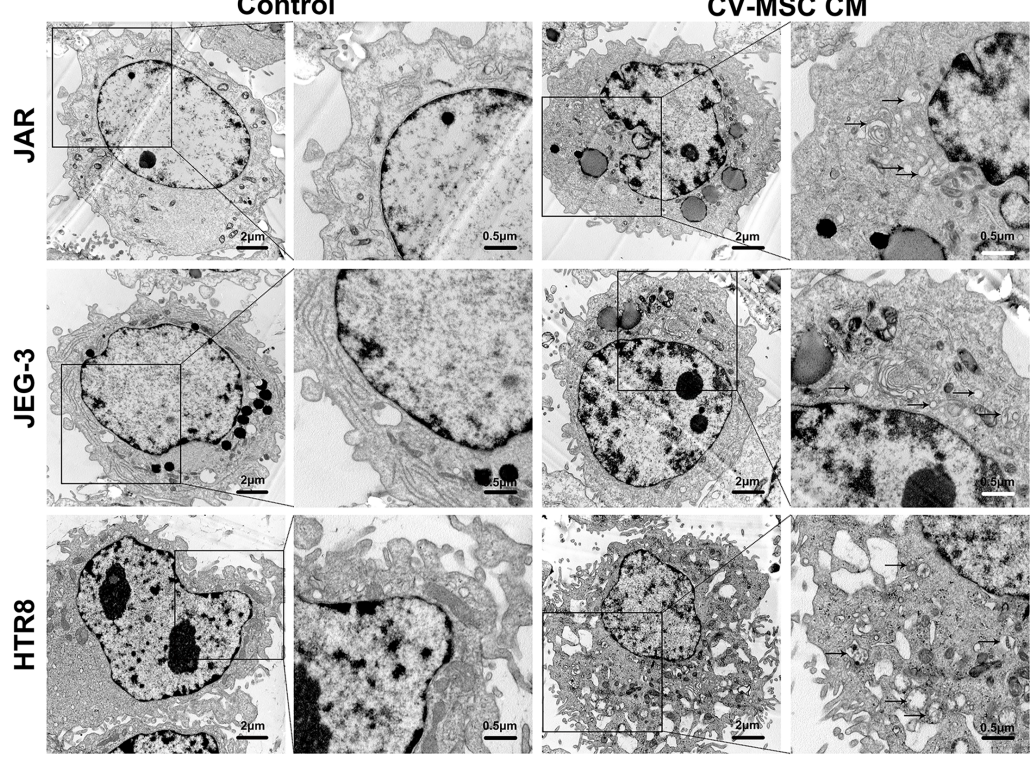

CV-MSC CM D

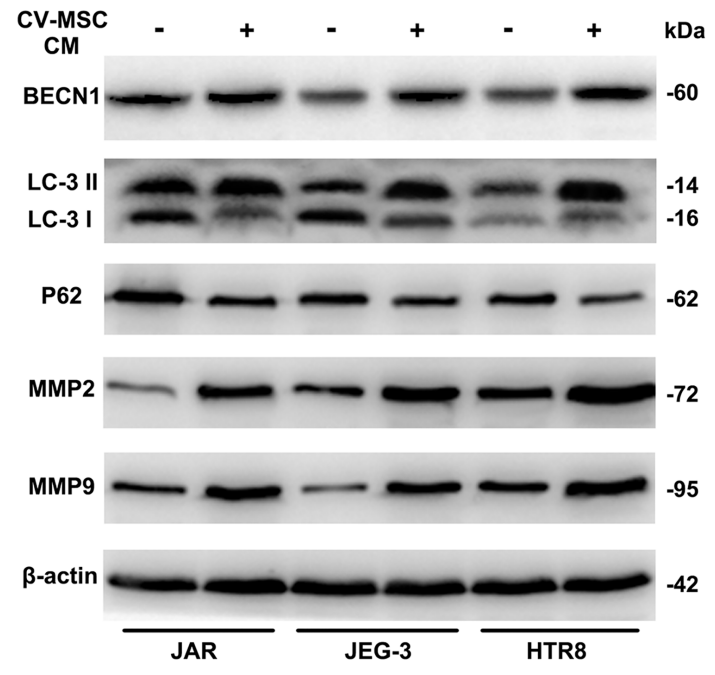




\section{Figure 1}

CV-MSCs promote the autophagy, proliferation and invasion of trophoblasts in vitro. (A) Representative results of CCK8 assays in JAR, JEG-3 and HTR-8 cells are shown. Trophoblast cells were treated with CVMSC CM under hypoxic condition. (B) Representative results of Transwell assays in trophoblasts are shown. The number of trophoblasts that migrated through the 8- $\mu \mathrm{m}$ Transwell membrane pores were counted to determine changes in the invasive capabilities in response to CV-MSC CM under hypoxic condition (scale bar, $50 \mu \mathrm{m}$ ). (C) Transmission electron microscopy (TEM) analysis of trophoblast cells showed autophagosome in the cytoplasm of the trophoblast cells treated with CV-MSC CM under hypoxia condition (scale bar, $1 \mu \mathrm{m}$ ). (D) Whole cell lysates from trophoblast cells were subjected to western blotting to analyze LC3, BECN1, p62, MMP2 and MMP9 levels. $\beta$-actin was included as a loading control. ${ }^{* \star *} \mathrm{P}<0.001$. 

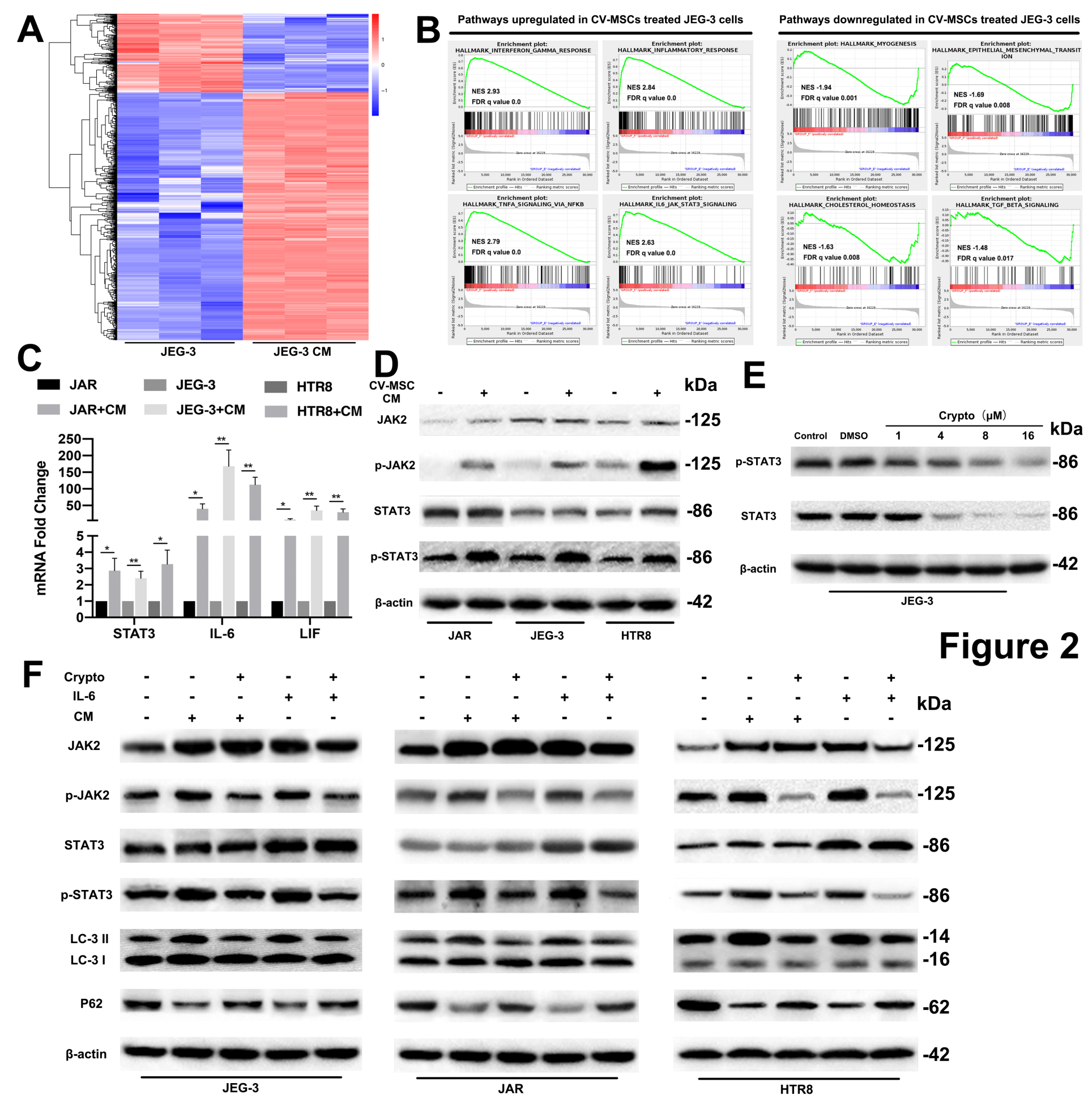
increases in p-JAK2 and p-STAT3 were found in JAR, JEG-3 and HTR8 cells treated with CV-MSC CM under hypoxic condition by western blotting. (E) Immunoblot analysis of STAT3 in JEG-3 cells treated with cryptotanshinone at the indicated concentration or DMSO as a control for 60 min before cultured in growth medium. (F) Three lines of trophoblast cells were cultured under hypoxia condition with or without cryptotanshinone, IL-6 or CV-MSC CM, and the expression of JAK2, p-JAK2, STAT3, p-STAT3, LC3 and P62 levels were tested by western blotting analysis. The results are expressed as the means \pm SD. $* \star \star$ $\mathrm{P}<0.001$.

A

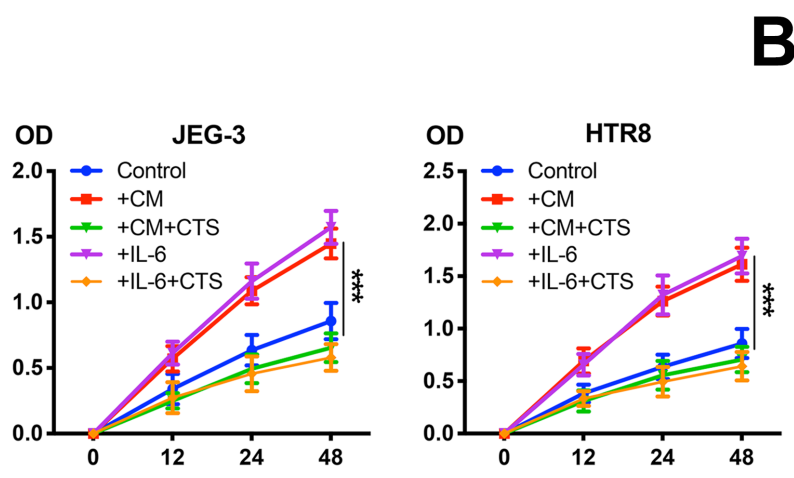

B
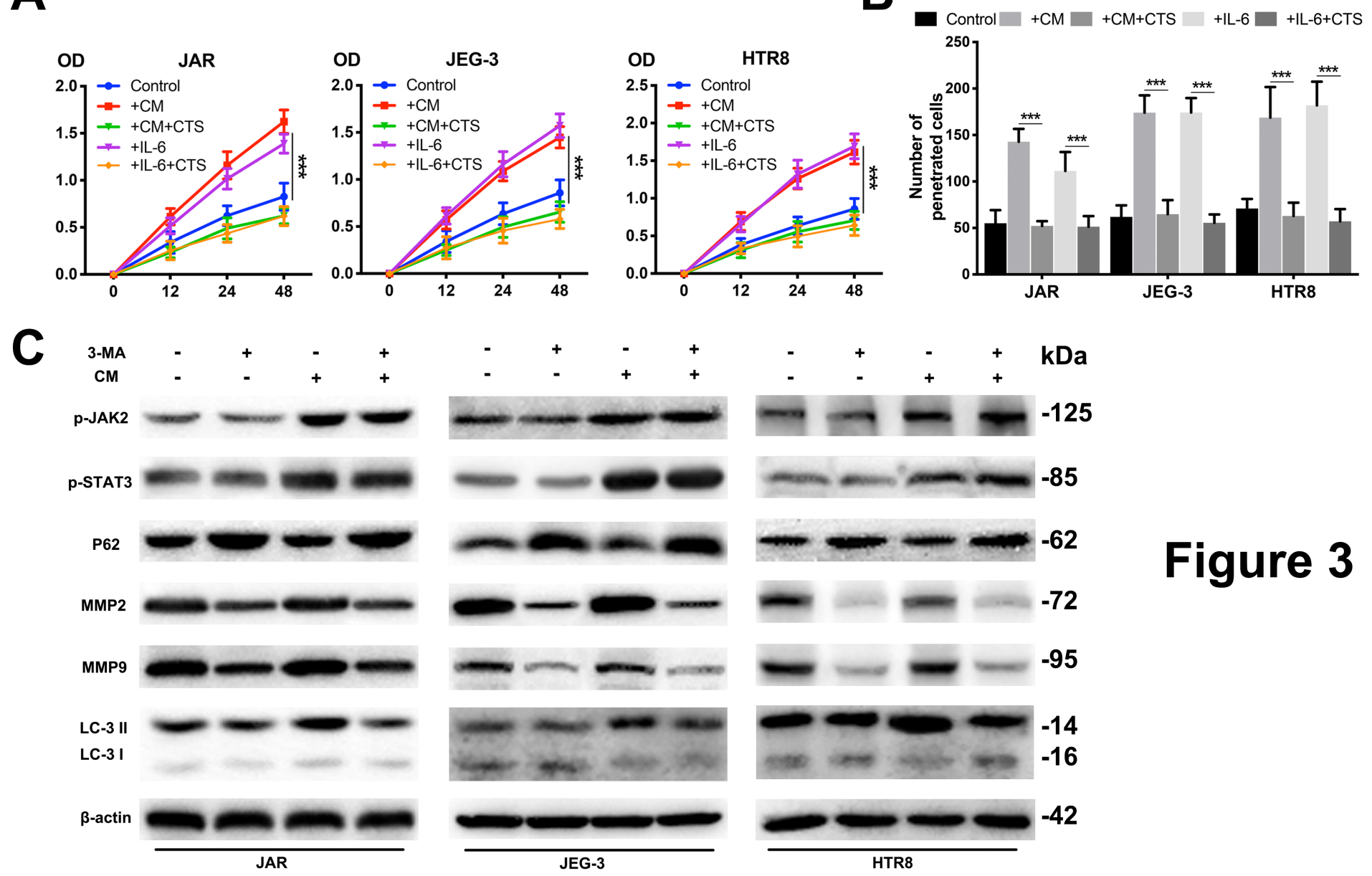

D
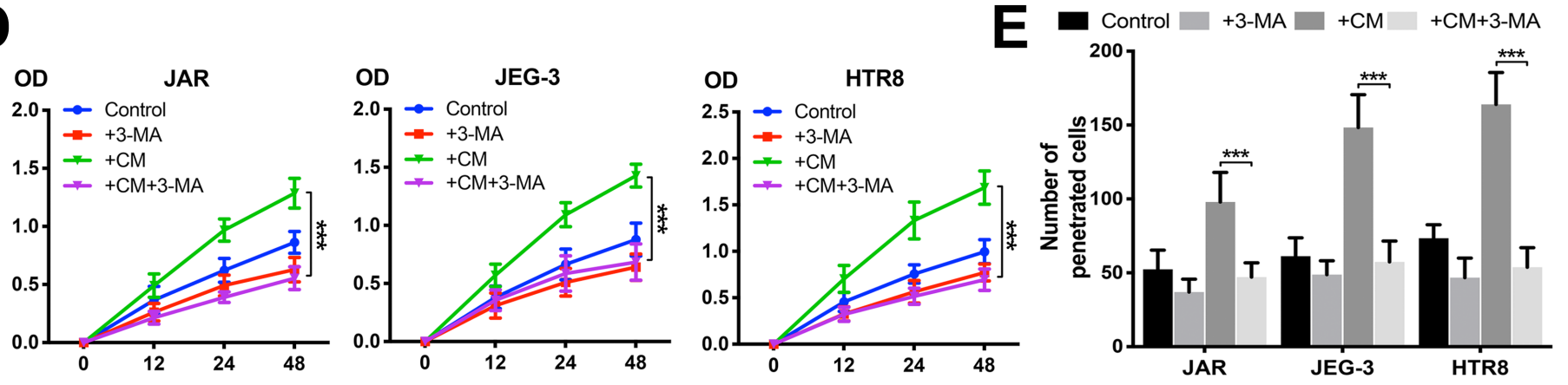

Figure 3 
CV-MSCs promoted trophoblasts growth and metastasis through JAK2/STAT3 signalling pathway. (A) Cell proliferation was evaluated by a CCK8 assay. Three trophoblast cell lines treated with were treated with IL-6 or CV-MSC CM with or without cryptotanshinone under hypoxic condition. Trophoblast cells cultured alone under hypoxic condition were served as control. (B) Trophoblast cells were treated with IL-6 or CV-MSC CM with or without cryptotanshinone, and invasion abilities were determined by Transwell assay. The number of penetrated cells were counted and statistical analysed. (C) Three lines of trophoblast cells were cultured under hypoxia condition with or without 3-MA or CV-MSC CM, and the expression of p-JAK2, p-STAT3, MMP2, MMP9 and LC3 levels were tested by western blotting analysis. (D) Cell proliferation was evaluated by a CCK8 assay. Three trophoblast cell lines treated with were treated with 3-MA or CV-MSC CM under hypoxic condition. Trophoblast cells cultured alone under hypoxic condition were served as control. (E) Trophoblast cells were treated with 3-MA or CV-MSC CM under hypoxic condition, and invasion abilities were determined by Transwell assay. The number of penetrated cells were counted and statistical analysed. ${ }^{* *} \mathrm{P}<0.001$.
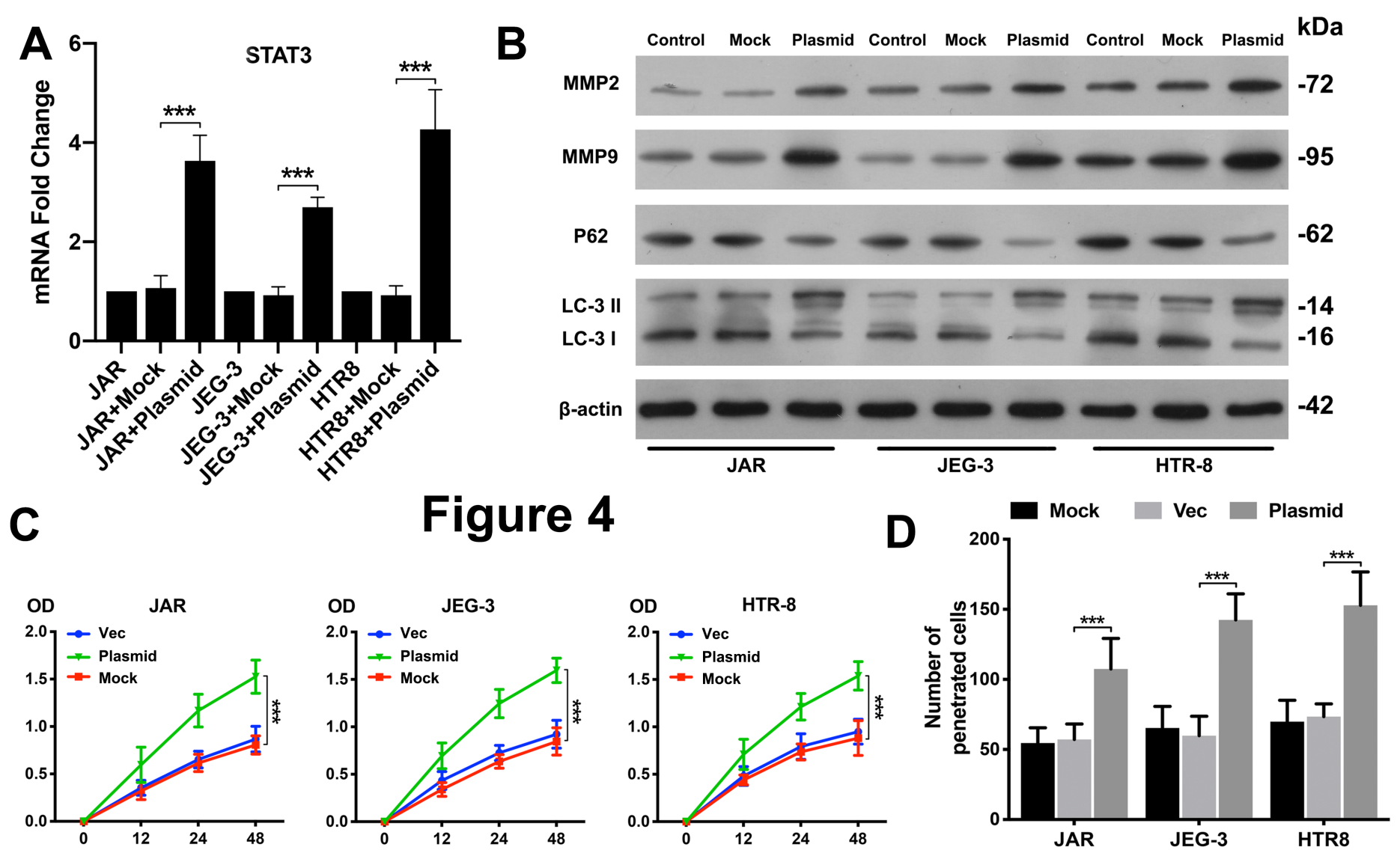

\section{Figure 4}

STAT3 regulates autophagy in trophoblast cells under hypoxia condition. (A) Significant increases in STAT3 mRNA were found in JAR, JEG-3 and HTR-8 cells transfected with STAT3 overexpression plasmid compared to untreated cells (Mock) or empty vector (Vec) under hypoxic condition by qRT-PCR. (B) Three lines of trophoblast cells were transfected with STAT3 overexpression plasmid under hypoxic condition, and the expression of MMP2, MMP9, P62 and LC3 levels were tested by western blotting analysis. 
Untreated trophoblast cells (Mock) and empty vector (Vec) cells were served as controls. (C) Cell proliferation was evaluated by a CCK8 assay. Three trophoblast cell lines were transfected with STAT3 overexpression plasmid or empty vector under hypoxic condition. Trophoblast cells cultured alone were served as control. (D) Trophoblast cells were transfected with STAT3 overexpression plasmid or empty vector under hypoxic condition, and invasion abilities were determined by Transwell assay. The number of penetrated cells were counted and statistical analysed. ${ }^{*} P<0.05$, ** $P<0.01$, *** $P<0.001$.
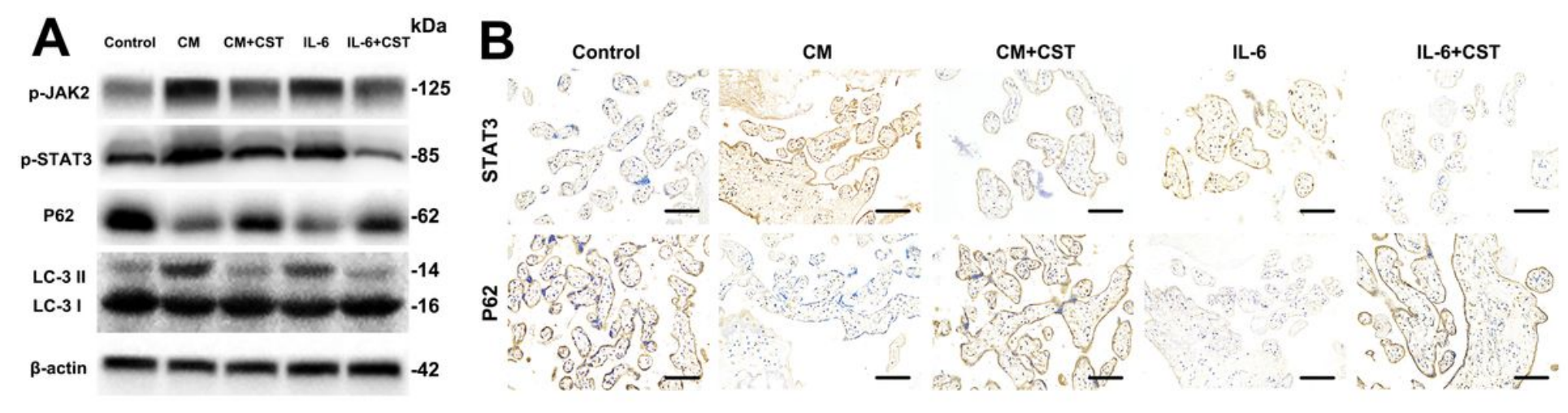

Figure 5

Figure 5

CV-MSC mediated STAT3 activation increased autophagy in placental explants under hypoxia condition. (A) The placental explants were treated with IL-6 or CV-MSC CM with or without cryptotanshinone under hypoxic condition, and the expression of p-JAK2, p-STAT3, P62 and LC3 levels were tested by western blotting analysis. Untreated placental explants under hypoxic condition were served as controls. (B) The placental explants were treated with IL-6 or CV-MSC CM with or without cryptotanshinone under hypoxic condition, and the expression of STAT3 and P62 levels were tested by Immunohistochemistry. Untreated placental explants were served as controls (scale bar, $50 \mu \mathrm{m}$ ).

\section{Supplementary Files}

This is a list of supplementary files associated with this preprint. Click to download.

- S3.tif

- S1.tif

- S2.tif 